\title{
The leucine zipper of TFE3 dictates helix-loop-helix dimerization specificity
}

\author{
Holger Beckmann and Tom Kadesch \\ Howard Hughes Medical Institute and Department of Human Genetics, University of Pennsylvania School of Medicine, \\ Philadelphia, Pennsylvania 19104-6148 USA
}

\begin{abstract}
TFE3 is a DNA-binding protein that activates transcription through the $\mu E 3$ site of the immunoglobulin heavy-chain enhancer. Its amino acid sequence reveals two putative protein dimerization motifs: a helix-loop-helix (HLH) and an adjacent leucine zipper. We show here that both of these motifs are necessary for TFE3 to homodimerize and to bind DNA in vitro. Using a dominant negative TFE3 mutant, we also demonstrate that both the HLH and the leucine zipper motifs are necessary and sufficient for protein-protein interactions in vivo. TFE3 is unable to form stable heterodimers with a variety of other HLH proteins, including USF, a protein that is structurally similar to TFE3 and binds a common DNA sequence. The analysis of "zipper swap" proteins in which the TFE3 HLH was fused to the leucine zipper region of USF indicates that dimerization specificity is mediated entirely by the identity of the leucine zipper and its position relative to the HLH. Hence, in this "b-HLH-zip" class of proteins, the leucine zipper functions in concert with the HLH both to stabilize protein-protein interactions and to establish dimerization specificity.
\end{abstract}

[Key Words: TFE3; USF; transcription factors; leucine zipper; helix-loop-helix; protein-protein interactions]

Received February 14, 1991; accepted March 8, 1991.

Many eukaryotic transcription factors have been shown to bind DNA as dimers or heterodimers (for review, see Johnson and McKnight 1989/. Two motifs that facilitate these protein-protein interactions have been defined thus far: the leucine zipper (Landschulz et al. 1988) and the helix-loop-helix (HLH; Murre et al. 1989a). Although distinct, each of these motifs is thought to mediate quaternary interactions through the hydrophobic faces of amphipathic $\alpha$-helices. For the case of the leucine zipper, an example of a coiled coil $/ \mathrm{O}^{\prime}$ Shea et al. 1989; Rasmussen et al. 1991), the hydrophobic face is defined in part by several leucine residues spaced every seventh amino acid ( $\mathrm{Hu}$ et al. 1990a and references therein). The HLH is thought to be comprised of two amphipathic helices separated by a loop of variable length and sequence. The leucine zipper and HLH motifs are generally situated adjacent to stretches of basic amino acids that are also required for DNA binding but not for protein-protein interactions (Benezra et al. 1990; Davis et al. 1990; Voronova and Baltimore 1990). Proteins that possess these motifs together are often referred to as b-zip and b-HLH proteins, respectively. Evidence favors a "scissors grip" model or an induced helical fork model for the binding of b-zip protein dimers to DNA (Vinson et al. 1989; O'Neil et al. 1990), which results in a net increase in the $\alpha$-helical content of the proteins (O'Neil et al. 1990; Patel et al. 1990; Talanian et al. 1990; Weiss et al. 1990). Although they are conceptually analogous to b-zip proteins, little is known concerning the precise structure of b-HLH proteins or how they bind DNA.
An additional, distinct class of proteins is defined by those that possess adjacent $\mathrm{HLH}$ and leucine zipper motifs. The leucine zipper in these proteins is generally found immediately carboxy-terminal to helix 2 of the HLH domain (referred to herein as HLH helix 2). Initially exemplified by $c-m y c$, as well as $\mathrm{N}-m y c$ and L-myc, members of this class of "b-HLH-zip" proteins include the mammalian transcription factors TFE3 (Beckmann et al. 1990), USF (Gregor et al. 1990), TFEB (Carr and Sharp 1990), and AP-4 (Hu et al. 1990b).

Here, we present a functional analysis of the b-HLHzip region of TFE3, a transcription factor that binds to, and activates transcription through, the immunoglobulin enhancer $\mu \mathrm{E} 3$ motif. We show that both the HLH and the leucine zipper of TFE3 are necessary for protein function and that the leucine zipper plays a critical role in defining interaction specificity among this class of HLH proteins.

Results

The TFE3 leucine zipper is required for DNA-binding activity

We synthesized a series of altered TFE3 proteins to assess the contribution of the leucine zipper to the DNAbinding activity of TFE3 in vitro (Fig. 1a). $\beta G-\lambda 3$ represents the coding sequence of the TFE3 cDNA linked to the $5^{\prime}$-untranslated region of the $\beta$-globin gene (including the $\beta$-globin ATG) and thus represents the intact TFE3 protein. $\beta \mathrm{G}-\lambda 3 \Delta 4$, for example, corresponds to a carboxy- 

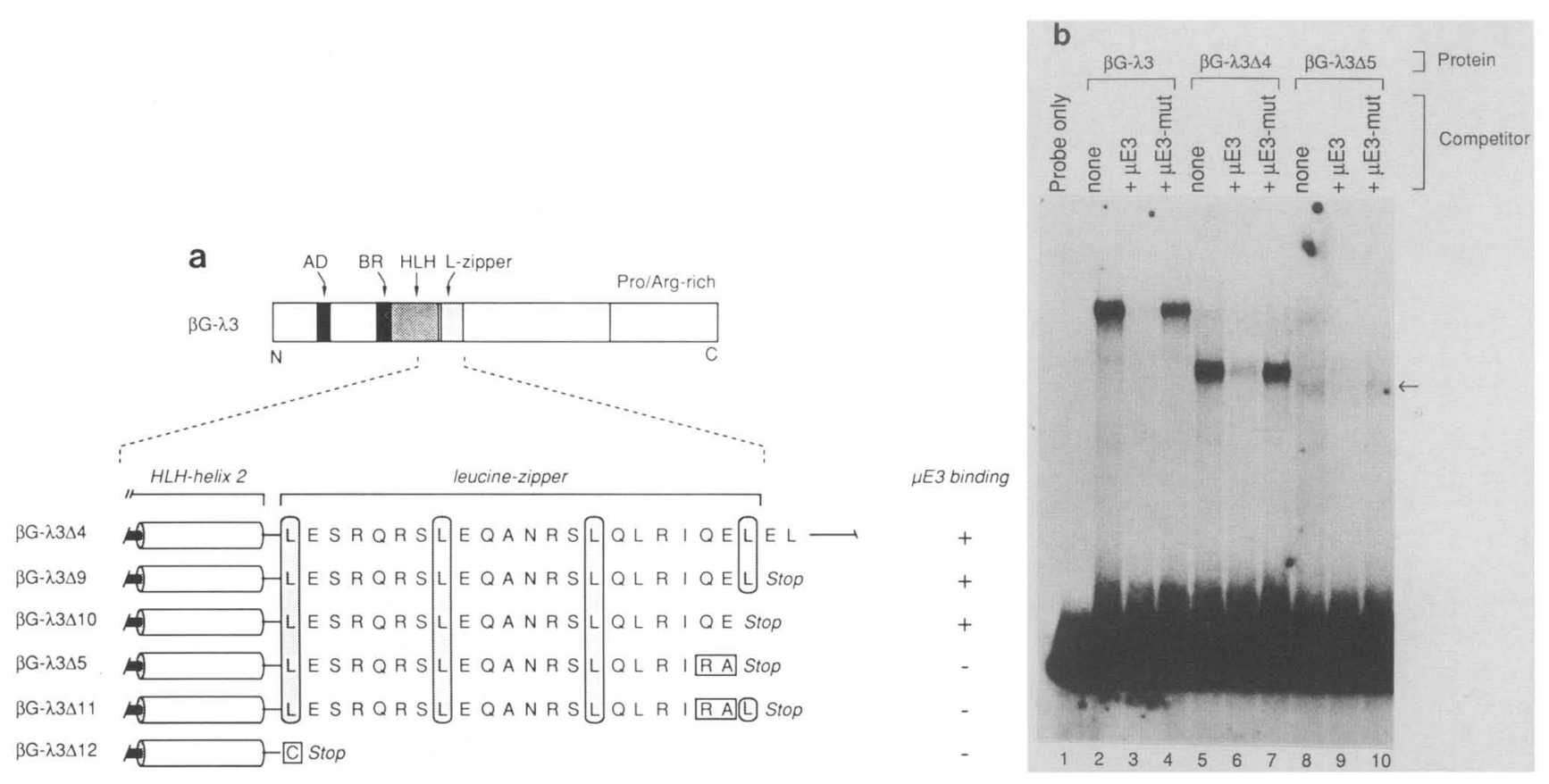

Figure 1. The leucine zipper of TFE3 is required for efficient DNA binding. (a) Schematic representation of the intact TFE3 protein $(\beta \mathrm{G}-\lambda 3)$ and summary of $\mu \mathrm{E} 3$-binding data for the indicated leucine zipper mutants. The transcriptional activation domain (AD), basic region (BR), helix-loop-helix $(\mathrm{HLH})$, and leucine zipper are indicated in the context of the full-length protein, and the amino acid sequence of the leucine zipper regions within the various protein forms tested are shown. A plus sign $(+\mid$ signifies $>75 \%$ wild-type binding affinity, and a minus sign $(-)$ indicates $<10 \%$ wild-type binding affinity. $(b)$ Representative mobility-shift assay using in vitro-synthesized TFE3 proteins. Plasmids or DNA fragments encoding the intact and deleted forms of TFE3 indicated were transcribed by T7 RNA polymerase, and RNA products were translated using reticulocyte lysates. Labeled DNA probe carrying a $\mu$ E3 site corresponds to IgH enhancer fragment 12 . Competitor DNAs consisted of oligonucleotides bearing normal ( $\mu$ E3) or mutant ( $\mu$ E3-mut) binding sites. The arrow indicates the position of a weakly shifted complex due to the $\beta G-\lambda 3 \Delta 5$ protein.

terminal truncation that leaves both the HLH and the leucine zipper intact. $\beta G-\lambda 3 \Delta 5$ is a carboxy-terminal truncation that removes the terminal leucine of the leucine zipper and introduces two amino acid substitutions. When these three proteins were synthesized in vitro, incubated with labeled DNA containing a $\mu \mathrm{E} 3$ site, and the complexes were analyzed using a mobility-shift assay, only $\beta G-\lambda 3$ and $\beta G-\lambda 3 \Delta 4$ were found to bind DNA efficiently (Fig. $1 b$ ). The $\beta G-\lambda 3 \Delta 5$ protein displayed extremely weak DNA binding, although it was synthesized in comparable amounts to the other two proteins (data not shown). TFE3 proteins that carry additional alterations in and around the terminal leucine were also tested, and the results are summarized in Figure la. Although the terminal leucine itself is not absolutely necessary (see $\beta G-\lambda 3 \Delta 10$ ), the two charged amino acids immediately preceding it are necessary (these may be involved in the formation of favorable salt bridges; Landschulz et al. 1988). We conclude that the four leucines adjacent to helix 2 of the HLH define the boundaries of a functional leucine zipper that is required for DNA binding.

\section{Oligomerization properties of TFE3}

The DNA-binding form of TFE3 was determined by cotranslating in vitro a full-length $(\beta G-\lambda 3)$ and a short- ened version of the protein $(\beta G-\lambda 3 \Delta 6$, containing only the b-HLH-zip region; see Materials and methods) and examining the DNA-binding products with a mobilityshift assay. As shown in Figure 2a, cotranslation of the two forms uniquely produces a shifted complex of intermediate mobility (lane 4), consistent with its identity as a heterodimer made up of the short and long versions of the protein. When the proteins were translated separately and mixed and incubated without DNA for up to $1 \mathrm{hr}$ at room temperature, no heterodimers were formed (data not shown). These results suggest that TFE3 dimers are very stable and do not exchange rapidly, and contrast the findings obtained with the b-zip protein C/EBP /Shuman et al. 1990).

Although deletions extending beyond the carboxy-terminal leucine of the TFE3 leucine zipper destroy DNA binding, we investigated the possibility that these mutant proteins may be able to form heterodimers with an intact TFE3 polypeptide in the presence of DNA. Cotranslation of intact TFE3 ( $\beta G-\lambda 3)$ and the leucine zipper mutant $(\beta G-\lambda 3 \Delta 5)$ did not result in the formation of any DNA-bound complexes other than those seen with translation of intact TFE3 alone (data not shown). Hence, both partners of the TFE 3 dimer must possess a functional leucine zipper.

Glutaraldehyde cross-linking of in vitro-translated TFE3 was carried out to further examine the relationship 

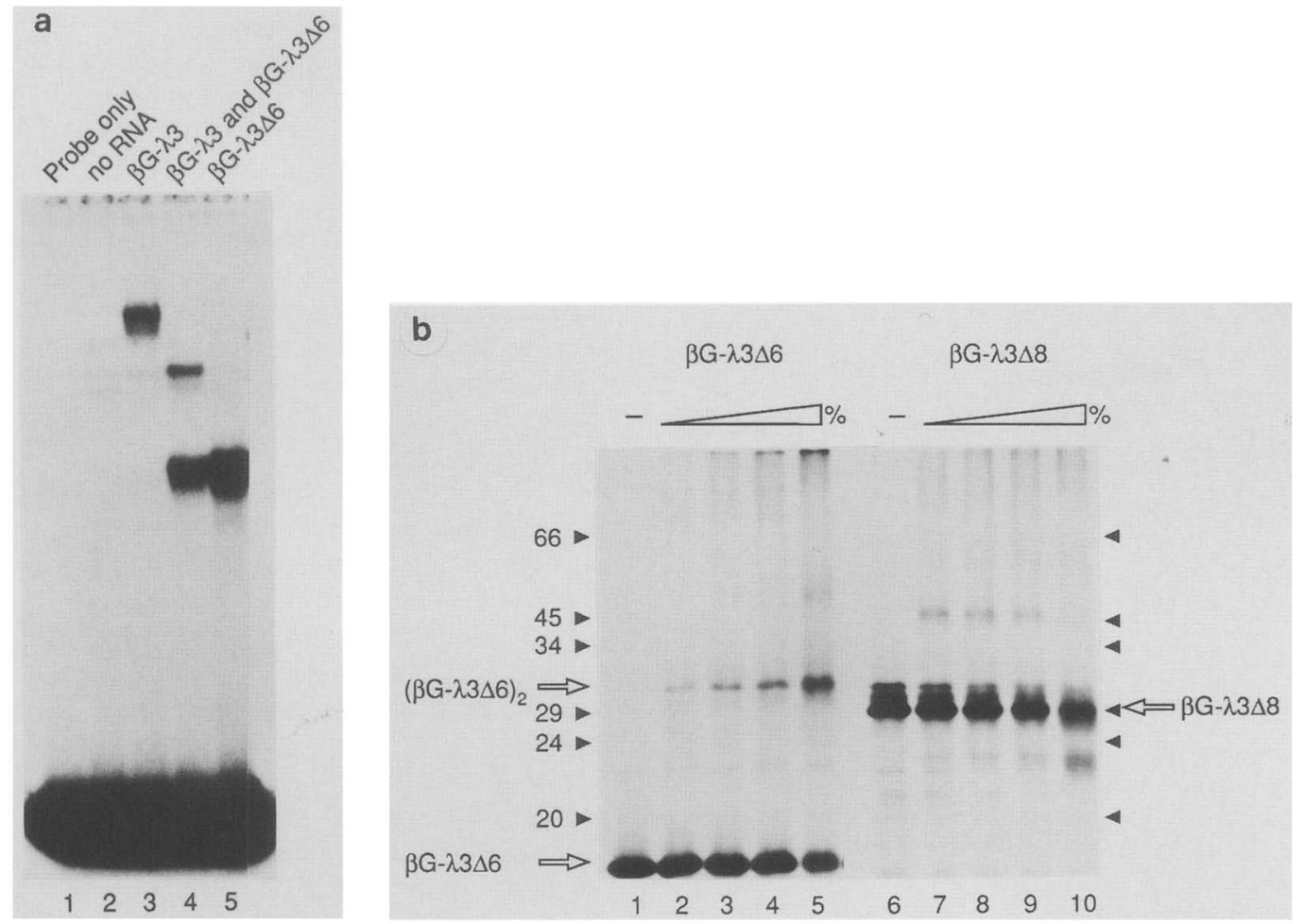

Figure 2. The leucine zipper of TFE3 is required for protein dimerization. (a) TFE3 binds DNA as a dimer. Intact ( $\beta$ G- $\lambda 3$ ) or truncated forms ( $\beta G-\lambda 3 \Delta 6$; see Materials and methods) of TFE3 were translated separately (lanes 3 and 5) or cotranslated (lane 4) and analyzed by a mobility-shift assay. Lane 2 (no RNA) indicates a binding reaction using reticulocyte lysate alone. (b) Glutaraldehyde cross-linking of in vitro-translated TFE 3 proteins. ${ }^{35}$ S-Labeled TFE3 proteins containing the b-HLH plus leucine zipper $(\beta G-\lambda 3 \Delta 6$; lanes $1-5)$ or b-HLH without the leucine zipper $(\beta \mathrm{G}-\lambda 3 \Delta 8$; lanes 6-10) were translated in vitro, treated with increasing concentrations of glutaraldehyde, and resolved by SDS-PAGE. Molecular weight standards are indicated by numbers and arrows. The positions of the $\beta G-\lambda 3 \Delta 6$ and $\beta G-\lambda 3 \Delta 8$ monomers are indicated, along with the $\beta G-\lambda 3 \Delta 6$ dimers. Longer exposure of the autoradiogram failed to reveal bands in the position expected of $\beta \mathrm{G}-\lambda 3 \Delta 8$ dimers. Final concentrations of glutaraldehyde were as follows: (Lanes 1 and 6 ) $0 \%$; (lanes 2 and 7) $0.0001 \%$; (lanes 3 and 8 ) $0.0002 \%$; (lanes 4 and 9) $0.0003 \%$; (lanes 5 and 10 ) $0.0004 \%$.

of DNA binding to its oligomeric structure. As shown in Figure $2 b$, treatment of $\beta G-\lambda 3 \Delta 6$ with glutaraldehyde resulted in the appearance of an additional protein species that migrated at a position expected of the protein dimer (lanes 2-5). No higher molecular weight species were observed with increasing concentrations of glutaraldehyde (Fig. 2b and data not shown). Treatment of in vitrotranslated $\beta G-\lambda 3 \Delta 8$, a form of TFE3 that lacks the entire leucine zipper (see Materials and methods), with similar (Fig. 2b, lanes 7-10) or higher (data not shown) concentrations of glutaraldehyde, failed to reveal the existence of protein dimers. Hence, the leucine zipper of TFE 3 is required both for DNA binding and for dimerization in solution.

Both the HLH and zipper motifs are necessary and sufficient for protein-protein interactions in vivo

We have previously mapped a major transcription activation domain within TFE3 by testing the ability of fusion proteins containing the first 147 amino acids of yeast GAL4 to activate minimal promoters containing GAL4-binding sites (Beckmann et al. 1990). We noted at the time of those studies that many proteins capable of stimulating transcription from GAL4-minimal promoters were unable to activate $\mu \mathrm{E} 3$-minimal promoters, although they possessed their HLH regions intact. Presumably, these proteins assumed a conformation that prevented them from binding a $\mu E 3$ motif in vivo. Remarkably, however, in cotransfection assays, those same GAL4 : TFE3 fusion proteins were found to inhibit the stimulatory activity of intact TFE 3 mediated through a cognate $\mu \mathrm{E} 3$ site (see below). Repression in such an assay is likely due to the formation of functionally inactive heterodimers made up of GAL4 : TFE3 fusion proteins and intact TFE3. This interpretation is formally analogous to that made with dominant-negative mutants of CREB, jun, and c-myc (Dang et al. 1989; Smeal et al. 1989; Dwarki et al. 1990), where heterodimer-mediated inhibition has also been demonstrated. We therefore made use of our experimental observation to map the regions of the TFE3 protein that mediate trans-dominant repression in vivo when linked to GAL4.

The effects of various GAL4 : TFE3 fusion proteins on the activity of intact TFE3 are shown in Figure 3. When a plasmid expressing intact TFE 3 was cotransfected into $\mathrm{NIH} / 3 \mathrm{~T} 3$ cells with a CAT reporter plasmid, using a 


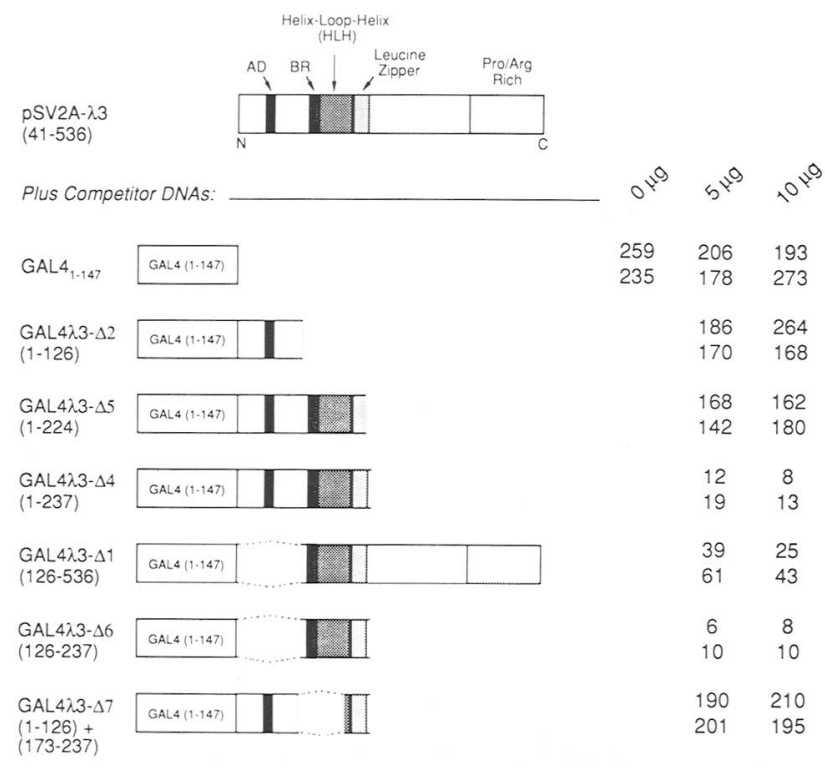

Figure 3. Trans-dominant repression of TFE3 in vivo by GAL4 : TFE3 fusion proteins requires the HLH and leucine zipper motifs. NIH/3T3 cells were transfected with $5 \mu \mathrm{g}$ of pSV2A 3 (expressing intact TFE 3 from the SV40 early promoter $], 1 \mu \mathrm{g}$ of $[\mu \mathrm{E} 3]_{4}-\mathrm{TATAcat}$ (a reporter that expresses CAT from a minimal promoter carrying four $\mu \mathrm{E} 3$ sites), and various plasmids $(5$ or $10 \mu \mathrm{g}$ ) that express the indicated GAL4 : TFE3 fusion proteins. In addition, cells were transfected with $5 \mu \mathrm{g}$ of $\mathrm{pCH} 110$ (a $\beta$-galactosidase expression plasmid) to normalize transfection efficiencies. The TFE 3 amino acids linked to the GAL4 DNA-binding domain [GAL4(1-147)] are indicated and are diagramed schematically relative to the intact TFE3 protein (shown at the top). Numerical values represent the relative levels of CATase obtained from two separate transfections. CATase activities obtained in the absence of the GAL4 plasmids are indicated in the column denoted $0 \mu \mathrm{g}$ competitor.

minimal promoter carrying $\mu \mathrm{E} 3$ sites, high levels of CATase activity were obtained (Fig. 3; $0 \mu$ g competitor). This activity was unaffected by cotransfecting additional plasmids that expressed GAL4 sequences alone (GAL4 147). However, cotransfection of any plasmid that expressed GAL4 linked to both the HLH and the leucine zipper motifs of TFE 3 (e.g., GAL $4 \lambda 3 \Delta 6$, GAL $4 \lambda 3 \Delta 1$, and GAL4 $\lambda 3 \Delta 4$ ) was sufficient to repress completely TFE3mediated CATase activity. Fusion proteins that carry deletions of the terminal leucine of the zipper (GAL4 $\lambda 3-\Delta 5$ ) or of the HLH (GAL4 $\lambda 3-\Delta 7$ ) failed to repress. These results indicate that trans-dominant repression requires, in addition to GAL4 sequences, both the HLH and the leucine zipper motifs of TFE3. We conclude that repression is due to the formation of inactive heterodimers and, hence, that both the HLH and the leucine zipper are necessary and sufficient for interactions of TFE3 monomers in vivo.

\section{The leucine zipper dictates dimerization specificity}

We have shown previously that TFE3 binds to the USFbinding site within the adenovirus major late promoter
(Beckmann et al. 1990; the $\mu \mathrm{E} 3$ and USF sites are identical at 10 of 11 positions). A cDNA encoding USF has recently been isolated, and the predicted amino acid sequence indicates that it is highly related to TFE3/Gregor et al. 1990). As with TFE3, USF has a leucine zipper situated carboxy-terminal to its HLH domain. Given the similar structure and DNA-binding specificity of the two proteins, we sought to determine whether they could form heterodimers. Accordingly, TFE3 and USF cDNAs were transcribed and translated in vitro and the resulting $\mu E 3$-binding activities were analyzed by a mobility-shift assay. As shown in Figure $4 b$, a short version of USF (containing amino acids 181-310) expressed alone was able to bind the $\mu \mathrm{E} 3$ element (lane 7$)$. However, cotranslation of USF and TFE3 did not result in any complexes of intermediate mobility that would have suggested the formation of heterodimers (lane 6). Similar results were obtained with a USF probe (data not shown). In separate experiments (data not shown) we have also been unable to demonstrate any dimerization of TFE3 with E2-5 (ITF-1), a b-HLH protein that contains the E47 HLH (Henthorn et al. 1990a,b), and of TFE3 with Id, a HLH protein that interacts with a variety of b-HLH proteins, including myoD, E12, and E47 (Benezra et al. 1990; M. Kiledjian, R. Benezra, P. Zwollo, S.M. Dymecki, S.V. Desiderio, and T. Kadesch, in prep.). We conclude that TFE3 does not promiscuously heterodimerize with other $\mathrm{HLH}$ proteins.

Because TFE3 and USF both contain leucine zippers, we sought to explore the generality of the hypothesis suggested by Tjian and co-workers (on the basis of their work with AP4; $\mathrm{Hu}$ et al. 1990b) that HLH-proximal leucine zippers function to dictate heterodimer specificity. We therefore performed a series of "zipper swap" experiments with TFE3 and USF. Initially, we synthesized a hybrid protein, designated $\beta G-\lambda 3$ : USF-1, that contains the b-HLH of TFE3 fused to the leucine zipper region of USF (the crossover was made at the last leucine in $\mathrm{HLH}$ helix 2 such that all residues amino-proximal are from TFE 3 and those carboxy-proximal, including the leucine zipper, are from USF; Fig. 4a). As shown in Figure $4 \mathrm{~b}$ (lanes $10-14$ ), $\beta \mathrm{G}-\lambda 3$ : USF-1 has the ability to bind a $\mu E 3$ oligonucleotide as an apparent homodimer (lane 12). When this hybrid protein was cotranslated with a truncated version of TFE3 ( $\beta G-\lambda 3 \Delta 6$; lane 11$)$, no complexes of intermediate mobility were seen, suggesting that the USF leucine zipper region prevents dimerization with TFE3. However, when $\beta G-\lambda 3$ : USF-1 was cotranslated

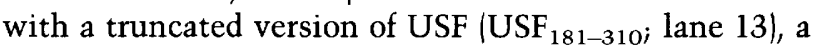
complex of intermediate mobility was observed, indicating the formation of heterodimers. Hence, dimerization specificity is dictated by the identity of the leucine zipper region and not by the HLH.

We considered the possibility that heterodimer formation between USF and TFE3 may be prevented simply by the different positions of their leucine zippers relative to HLH helix 2. Four of the b-HLH-zip proteins characterized thus far have a distinct spacing between the last conserved amino acid of HLH helix 2 and the first leucine of their leucine zippers (see Fig. 6, below). Interest- 
a

$$
\begin{aligned}
& (1-233) \\
& \beta G-\lambda 3 \Delta 6 \\
& (127-233) \\
& U S F \\
& (181-310)
\end{aligned}
$$

$\beta G-\lambda 3: U S F-1$
$(1-192 / 254-310)$

$\beta G-\lambda 3: U S F-2 L$

(1-201/271-310)

$\beta G-\lambda 3: U S F-2 S$

(127-201/271-310)

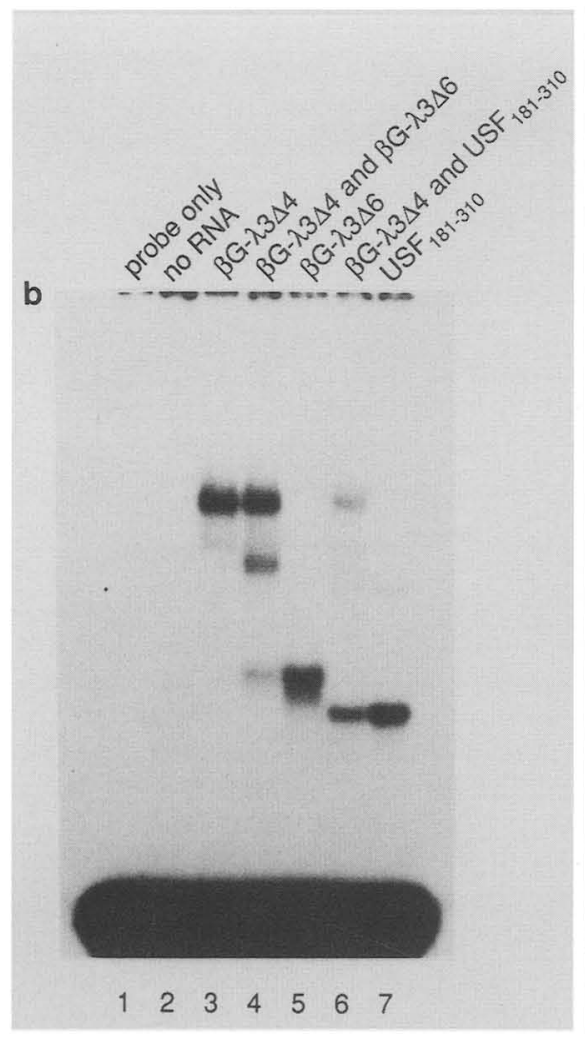

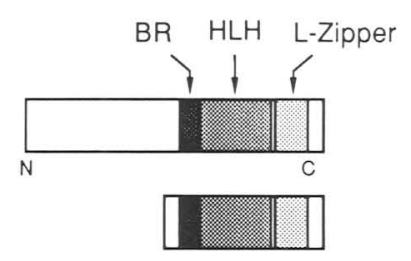
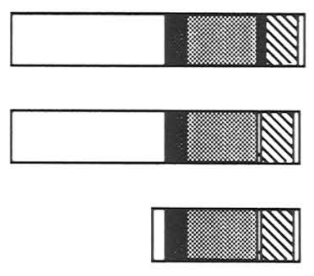
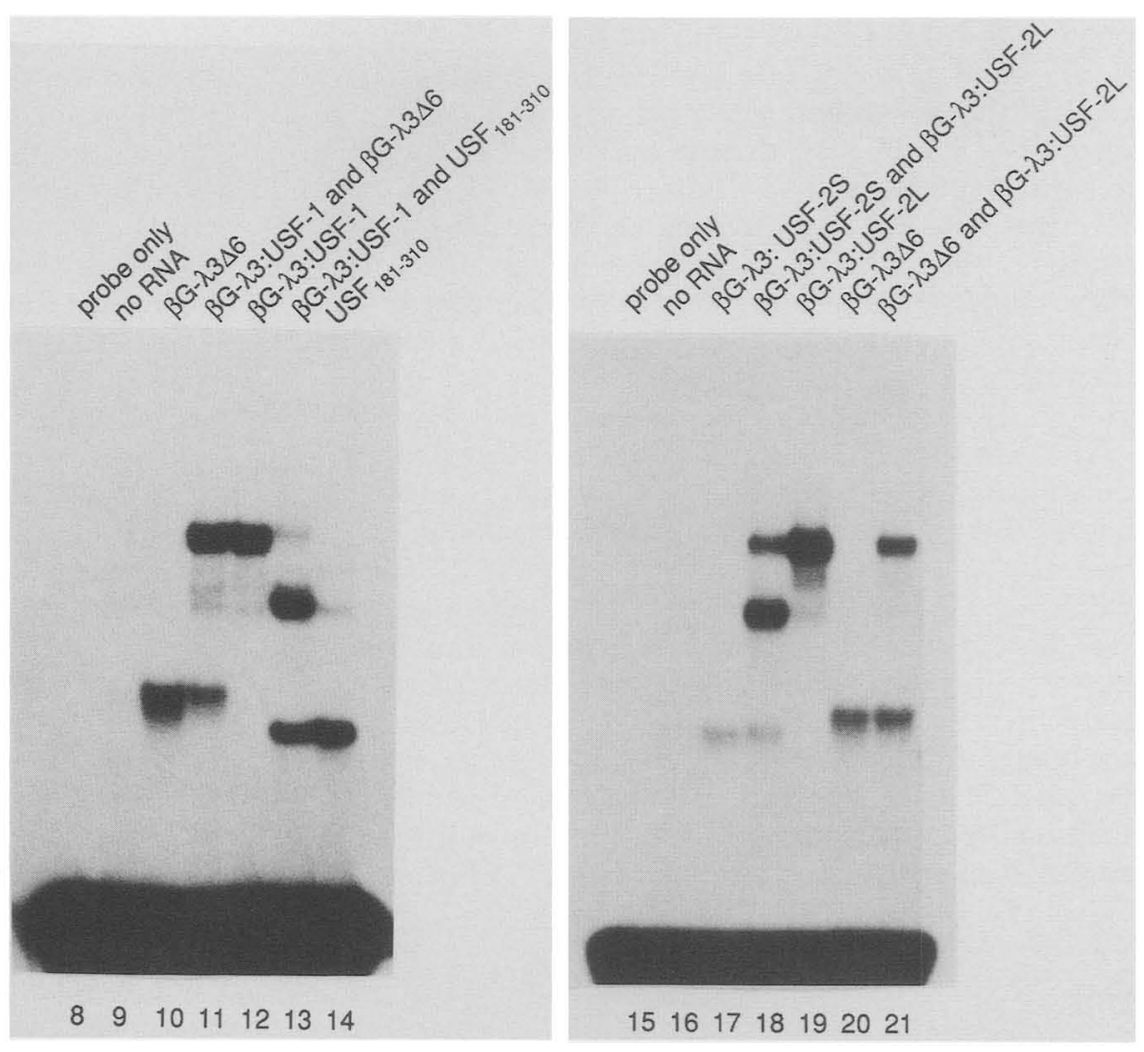

Figure 4. The dimerization specificities of TFE 3 and USF are dictated by the position and identity of their leucine zippers. (a) Schematic representation of proteins used in these experiments. The basic motifs (BR), helix-loop-helix (HLH), and leucine zipper regions of TFE3 and USF are indicated (details of each protein are given in Materials and methods). (b) Lanes 1-7 (left), lanes 8-14 (middle), and lanes 15-21 (right) indicate results of binding reactions using a $\mu \mathrm{E} 3$ probe and proteins generated in vitro using reticulocyte lysates. (Lanes 1,8, and 15) Probe only; (lanes 2, 9, and 16) binding reactions using reticulocyte lysates alone. Other individual translations or cotranslations with the various protein forms are as indicated. (Left) TFE3 and USF do not heterodimerize. TFE3 ( $\beta G-\lambda 3 \Delta 4$ or $\beta G-\lambda 3 \Delta 6$ ) and USF (amino acids 181-310; Gregor et al. 1990) were translated separately or together, as indicated. Novel bands specific to cotranslation reactions were only observed in lane 4 ( $\beta G-\lambda 3 \Delta 4$ plus $\beta G-\lambda 3 \Delta 6$ ), even upon longer exposure of the autoradiogram. (Middle) A hybrid protein ( $\beta$ G- $\lambda 3$ : USF-1; see $a$ ) containing the TFE3 HLH and USF leucine zipper region is able to heterodimerize with USF but not with TFE3. Proteins were translated alone or together, as indicated. Intermediate bands specific to cotranslation reactions were limited to lane 13 in which $\beta G \lambda 3$ : USF-1 was cotranslated with USF. (Right) The USF leucine zipper is incompatible with the TFE3 leucine zipper. A long $(\mathrm{L})$ version of a fusion protein $(\beta \mathrm{G}-\lambda 3$ : USF- 2 ; see $a$ ) containing a precise replacement of the TFE3 leucine zipper with that of USF was translated alone (lane 19), together with a short (S) version of the same protein (lane 18), or with a truncated version of TFE3 ( $\beta G-\lambda 3 \Delta 6$; lane 21). Bands specific to cotranslation reactions were limited to lane 18 , in which the short and long versions of $\beta G-\lambda 3$ : USF- 2 were cotranslated. 
ingly, the positions of the leucine zippers differ by what may be integral numbers of $\alpha$-helical turns. These regions are predicted to be extended $\alpha$-helices /Chou and Fassman 1974), and this suggests that the hydrophobic surface of HLH helix 2 gradually precesses around the $\alpha$-helix to link up with the hydrophobic surfaces of the leucine zipper. Compared with TFE3, the USF leucine zipper begins 7 amino acids (two helical turns) farther away from HLH helix 2 and, hence, it may be difficult to align simultaneously both motifs in a heterodimer (i.e., in a TFE3/USF heterodimer or in a TFE $3 / \beta G-\lambda 3:$ USF-1 heterodimer, the USF amino acids that separate HLH helix 2 from the leucine zipper would have to loop out).

To test the importance of the spacing between HLH helix 2 and the leucine zipper, we synthesized an additional zipper swap protein, designated TFE3 : USF-2 (Fig. $4 a \mid$, which precisely replaces the TFE3 leucine zipper with that of USF, such that the distance between the leucine zipper and the HLH helix 2 in the hybrid protein is the same as that found in TFE3/the crossover was made at the first leucine of the respective leucine zippers of the two proteins). Although this hybrid protein was able to bind DNA as a homodimer (Fig. 4b, lanes 17 and 19; note also that cotranslation of short and long versions of TFE3 : USF-2 gave rise to intermediate complexes, lane 18), indicating that the leucine zipper was functioning, it was not able to form heterodimers with either TFE3 ( $\beta G-\lambda 3 \Delta 6$; lane 21 ) or USF (data not shown). Hence, the respective leucine zippers of USF and TFE3, even when similarly aligned, are unable to function with one another to stabilize HLH interactions. Similar incompatibilities have been noted for leucine zippers in the b-zip family of proteins (Kouzarides and Ziff 1989). TFE3 : USF-2 was also unable to heterodimerize with USF (data not shown). Hence, even given compatible leucine zippers, dimer formation requires them to be similarly aligned with respect to HLH helix 2. Preliminary experiments indicate that the particular amino acids between HLH helix 2 and the leucine zipper are not critical for dimer specificity (data not shown). The results of the zipper swap experiments are summarized in Figure 5.

\section{Discussion}

It has been shown recently that, as with TFE3, the transcription factors USF (Gregor et al. 1990) and AP-4 (Hu et al. 1990b/ contain both HLH and adjacent leucine zipper motifs. Although it is clear that the leucine zipper plays a role in stabilizing protein-protein interactions mediated by the HLH, the precise roles of these two motifs may be somewhat different for each protein. We find, for example, that the leucine zipper of TFE3 is absolutely required for DNA binding in vitro. Glutaraldehyde crosslinking experiments and in vivo inhibition studies correlate DNA binding with protein dimerization. For the case of USF, the leucine zipper is required for efficient DNA binding of the full-length protein but not of a truncated protein. Hence, for a truncated USF, the HLH is sufficient to establish dimer formation in the presence of DNA. AP-4 has two leucine zippers; one is adjacent to the HLH domain. If both zippers are deleted, the protein is unable to form dimers in solution but is able to bind DNA as a homodimer or as a heterodimer with the bHLH protein E12 (Hu et al. 1990b). Hence, for AP-4 and USF, the HLH may be sufficient to mediate protein-protein interactions but only in the presence of DNA. The HLH domain of TFE3, when separated from a functional leucine zipper, is completely incapable of mediating dimer formation, even in the presence of DNA.

$\mathrm{HLH}$ and leucine zipper motifs are generally thought to mediate protein-protein interactions through the hydrophobic surfaces of amphipathic $\alpha$-helices. Although the leucine zipper of TFE3 can be identified as a distinct protein dimerization motif, we have been unable to separate it functionally from the HLH. Neither motif alone is sufficient for the formation of stable protein-protein interactions when measured either in vitro or in vivo. An examination of HLH helix 2 of TFE 3 reveals both quantitative and qualitative differences in its hydrophobic nature compared with the corresponding helices of E12, E47 (E2-5), or E2-2. These latter b-HLH proteins lack, and therefore do not require, the stabilizing effects of adjacent leucine zippers. An examination of the leucine zipper of TFE3 indicates its poor adherence to the threefour rule that defines the hydrophobic "spine" of coiled coils (Hu et al. 1990a,b). Hence, the relatively poor hydrophobic character of these individual motifs (i.e., helix 2 and the leucine zipper of TFE3/ may explain why they must function together. Given the potential influences of nonhydrophobic residues (e.g., in the formation of stabilizing salt bridges), however, the parameters that govern the dimerization tendencies of these particular motifs may not be this straightforward.

Figure 5. Heterodimer specificities displayed by TFE3, USF, and TFE3 : USF hybrid proteins. Note that all of the indicated proteins bind DNA as apparent homodimers and, therefore, possess functional HLH and leucine zipper motifs.
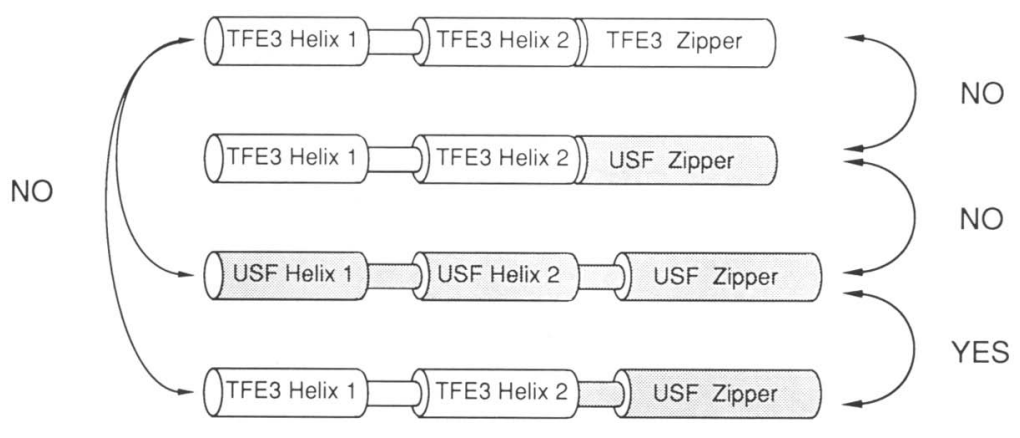
Unlike a variety of other HLH proteins, such as E12, E47, MyoD, myogenin, Id, and daughterless (Murre et al. 1989b; Benezra et al. 1990), TFE3 does not promiscuously heterodimerize. Although this distinction immediately points to a role for the leucine zipper in limiting the array of interactions among $\mathrm{HLH}$ proteins, the presence of the leucine zipper does not, by itself, define a separate group of heterodimerizing HLH proteins. This is most clearly exemplified by our finding that TFE 3 does not heterodimerize with USF, although both of these proteins contain adjacent HLH and leucine zipper motifs and can bind to a common DNA site. The idea that different leucine zippers specify distinct subsets of proteinprotein interactions is supported by our finding that the TFE3 : USF-1 hybrid protein (containing the TFE3 HLH and the USF leucine zipper region) is able to heterodimerize with USF but not with TFE3.

We have tested two possible explanations for how the leucine zipper region imparts dimerization specificity. One is suggested by the different spacing between HLH helix 2 and the leucine zipper found in the various bHLH-zip proteins (Fig. 6). Considering the close proximity of the leucine zipper to helix 2 in these proteins, it is possible that together they comprise an extended $\alpha$-helix (this is supported by Chou-Fassman predictions). Hence, when compared, the leucine zippers of most of the bHLH-zip proteins are positioned differently with respect to their HLH helix 2 motifs (i.e., separated by different integral numbers of $\alpha$-helical turns). Therefore, given this limited (but growing) set of proteins, interactions requiring an optimum alignment of the hydrophobic faces of HLH helix 2 and the leucine zipper would best be obtained with identical protein monomers. Our demonstration that USF does not form heterodimers with TFE3 : USF-2 (in which the USF leucine zipper is placed 7 amino acids closer to TFE3 HLH helix 2) confirms this idea. A second, nonexclusive explanation for the leucine zipper imparting HLH-mediated dimerization specificity is that the leucine zippers themselves may be incompatible. It has been established that b-zip proteins display restricted dimerization specificities due to the particular characteristics of their leucine zippers. Our observation that TFE3 is unable to heterodimerize with TFE3 : USF2 (in which the TFE3 leucine zipper is replaced exactly with that of USF) confirms that the leucine zippers of TFE 3 and USF are incompatible. Hence, both the position and identity of the leucine zippers dictate dimerization specificity in this family of proteins.

Although our results have specifically addressed the behavior of TFE3 and USF, we suspect that the rules governing their dimerization properties would also apply to other b-HLH-zip proteins. It has been shown that the HLH-proximal leucine zipper of AP4 restricts its ability to heterodimerize with other b-HLH proteins such as E12 (Hu et al. 1990b). We propose classifying b-HLH-zip proteins on the basis of the spacing between HLH helix 2 and the leucine zipper (see Fig. 6). Of the known members of this family, TFEB would be grouped with TFE3, L-myc would be grouped with c-myc, and N-myc would be grouped with AP4. Although this classification scheme may be of some predictive value in identifying possible partners for these proteins, it does not account for leucine zipper incompatibility and, hence, may represent only a useful first step. Moreover, interaction specificities may be further restricted by additional, more conventional leucine zippers within the proteins, such as that described in AP4 (Hu et al. 1990b).

\section{Materials and methods}

Plasmids

All plasmids were constructed and manipulated using standard techniques (Maniatis et al. 1982; Ausubel et al. 1987). Plasmids expressing the GAL4: TFE3 fusion proteins GAL $4 \lambda 3-\Delta 1$ and GAL4 $\lambda 3-\Delta 2$ have been described previously (Beckmann et al. 1990). The plasmid expressing GAL $4 \lambda 3-\Delta 4$ was generated by inserting an EcoRI-RsaI fragment from the $\lambda 3$ cDNA into pGAL4 $_{1-147}$ (Lillie and Green 1989) cut with EcoRI and SmaI. The GAL $4 \lambda 3-\Delta 5$ expression plasmid was constructed by inserting an EcoRI fragment from the $\lambda 3$ cDNA into pGAL4 $4_{1-147}$ cut
HLH Helix 2 Leucine Zipper

USF

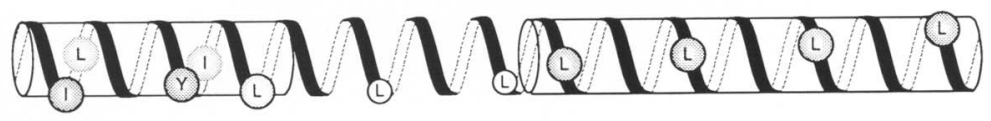

c-myc

$\left[\mathrm{L}-\mathrm{myc} c^{\star}\right]$

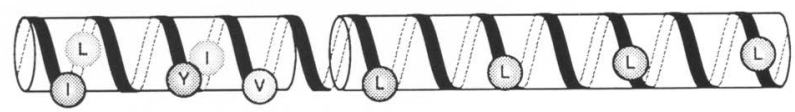

$\mathrm{AP}-4$

[N-myc]

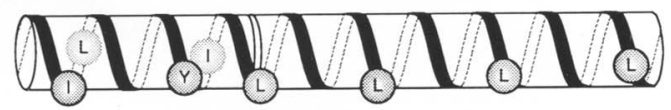

Figure 6. Position of the leucine zipper relative to HLH helix 2 in various b-HLH-zip proteins. (TFEB ${ }^{\star}$ ) Replace the final leucine of the TFE3 HLH helix 2 with a methionine; $\left(\mathrm{L}-m y c^{\star}\right)$ replace the final valine of the cmyc HLH helix 2 with a leucine and the first leucine of the c-myc leucine zipper with an alanine. 
with EcoRI. The plasmid expressing GAL $4 \lambda 3-\Delta 6$ was generated by inserting a $B g I I I-R s a I$ fragment from the $\lambda 3 \mathrm{cDNA}$ between the BamHI and SmaI sites of Gal4 ${ }_{1-147}$. The plasmid expressing GAL4 $\lambda 3-\Delta 7$ was constructed by replacing a $B g l I I-S t u I$ fragment containing the $\lambda 3$ cDNA of GAL $4 \lambda 3-\Delta 4$ with a BamHI-StuI synthetic polylinker (see below). Plasmid T7 $\beta G-\lambda 3 \Delta 4$ was constructed by replacing a $B g / I I-X b a I$ fragment of T7 $\beta G-\lambda 3$ (Beckmann et al. 1990) with a $B g l I I-X b a I$ fragment from GAL4 $43-\Delta 4$, containing the $\lambda 3 \mathrm{cDNA}$. The plasmid T7 $\beta \mathrm{G}-\lambda 3 \Delta 5$ was generated by replacing a $B g I I I-X b a I$ fragment of $T 7 \beta G-\lambda 3$ with a $B g l I I-X b a I$ fragment from GAL4 $\lambda 3-\Delta 5$, containing the $\lambda 3$ cDNA. Plasmid T7 $\beta G-\lambda 3 \Delta 8$ was generated by replacing a BglII$S c a I$ fragment from T7 $\beta$ G- $\lambda 3$ with a BglII-PstI fragment containing the $\lambda 3$ cDNA and a Pstl-Scal fragment from pGem4 (Promega). T7 $\beta$ G $-\lambda 3 \Delta 6$ and T7 $\beta$ G- $\lambda 3$ : USF expression DNA fragments were generated by the polymerase chain reaction (PCR). T7 $\beta G-\lambda 3 \Delta 6$ was PCR-generated by annealing an SP6 primer (Promega) and a primer (T7 PCR primer, see below) carrying, in series, the $T 7$ promoter sequence, 6 nucleotides of the 5 -untranslated region of the $\beta$-globin gene, an AUG, and 20 nucleotides corresponding to the $\lambda 3 \mathrm{cDNA}$, to a BglII-ScaI fragment containing the $\lambda 3$ cDNA from plasmid T7 $\beta G-\lambda 3 \Delta 4$. $T 7 \beta G-\lambda 3 \Delta 9, T 7 \beta G-\lambda 3 \Delta 10$, and T7 $\beta G-\lambda 3 \Delta 12$ were generated in PCR reactions containing the $T 7$ primer and primers PL-Stop, P-Stop, and C-Stop, respectively. The first two of these PCR products were cloned as HindIII- $\mathrm{XbaI}$ fragments into the plasmid T7 $\beta \Delta-6$ Sal (Norman et al. 1988). T7 $\beta$ G- $\lambda 3$ : USF-1 (used to synthesize protein $\beta G-\lambda 3$ : USF-1) was PCR-generated in several steps involving an overlap extension protocol (Horton et al. 1990). First, two separate products were generated using (1) the fE3 primer (see below) and the T7-PCR primer annealed to $T 7 \beta G-\lambda 3$, and (2) the fUSF primer (see below) and an SK primer (Stratagene) annealed to USF 181-310 (kindly provided by P. Gregor and R. Roeder; Gregor et al. 1990). Second, these two independently generated products were melted and annealed, and the complementary strands were extended in a single PCR cycle. Finally, this intermediate was annealed to the T7-PCR primer and SK primer. T7 $\beta G-\lambda 3$ : USF-2L and T7 $\beta G$ $\lambda 3$ : USF-2S /used to synthesize proteins TFE $3:$ USF-2L and TFE3 : USF-2S) were generated in a similar way. The initial two products were generated by using (1) a combination of either a T7 primer (T7 $\beta \mathrm{G}-\lambda 3: \mathrm{USF}-2 \mathrm{~L})$ or a $\mathrm{T} 7$ primer $(\mathrm{T} 7 \beta \mathrm{G}$ $-\lambda 3$ : USF-2S) and primer fE3-2 annealed to $T 7 \beta G-\lambda 3$ and (2) the fUSF- 2 primer and an SK primer annealed to USF 181-310. The final PCR products were used directly for in vitro transcription and translation reactions.

In constructing the expression plasmids described above, the following amino acids would be added to those directly encoded the various TFE 3 cDNA segments:

T7 $\beta G-\lambda 3 \Delta 4: \quad$ Gly-Asn-Ser-Ser linked carboxy-terminal to Pro $_{233}$

T7 $\beta G-\lambda 3 \Delta 5: \quad$ Arg-Ala linked carboxy-terminal to Ile 220

T7 $\beta$ G- $\lambda 3 \Delta 6$ : Gly-Asn-Ser-Ser-Ser-Arg-Leu-Arg-Gly-Glu-

Trp-Leu-Arg-Ala-His-Lys-Ile-Ser-Gln-TrpIle linked carboxy-terminal to $\mathrm{Pro}_{233}$

T7 $\beta G$ - $\lambda 3 \Delta 8$ : Tyr-Asp-Val-Ser linked carboxy-terminal to Gln 193

GAL4 $\lambda$ 3- $\Delta 4$ : Gly-Asn-Ser-Ser-Ser-Arg linked carboxy-terminal to $\mathrm{Pro}_{233}$

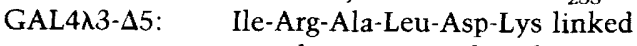
carboxy-terminal to $\mathrm{Ile}_{220}$

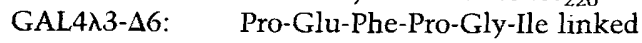
amino-terminal to $\mathrm{Ile}_{126}$ and Gly-Asn-Ser-Ser-Ser-Arg linked carboxy-terminal to $\mathrm{Pro}_{233}$

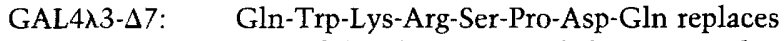
the deleted TFE3-encoded amino acids (between $\mathrm{Ile}_{126}$ and $\mathrm{Arg}_{183}$ ) and Gly-Asn-Ser-Ser-Ser Arg linked carboxy-terminal to $\mathrm{Pro}_{233}$

\section{Synthetic oligonucleotides}

The oligonucleotides listed in Table 1 were synthesized by the University of Pennsylvania Cancer Center and by the Howard Hughes Medical Institute, respectively.

In vitro transcription and translation reactions

In vitro transcription and translation reactions were carried out as described previously (Beckmann et al. 1990). For cotranslation experiments approximately equal amounts of in vitro-generated RNAs were added to the translation mix.

\section{Mobility-shift assays}

DNA probes were prepared by labeling IgH enhancer fragments or $\mu \mathrm{E} 3$ oligonucleotides by filling 5 ' overhangs with DNA polymerase (Klenow, Promega) and deoxyribonucleoside triphosphates $\left[\alpha-{ }^{32} \mathrm{P}\right] \mathrm{dATP}$ or $\left[\alpha^{-{ }^{32}} \mathrm{P}\right] \mathrm{dGTP}$. Isolation of IgH enhancer fragment 12 , containing a single $\mu \mathrm{E} 3$ site, and binding reactions were carried out as described previously (Beckmann et al. 1990).

\section{Chemical cross-linking}

In vitro-generated $\left[{ }^{35} S\right]$ methionine-labeled proteins were diluted $1: 10$ in buffer $\mathrm{R}[0.1 \mathrm{M} \mathrm{KCl}, 20 \mathrm{~mm}$ HEPES $(\mathrm{pH} 7.9), 20 \%$ glycerol, $0.2 \mathrm{mM}$ EDTA, $0.5 \mathrm{~mm}$ DTT]. Glutaraldehyde (diluted in buffer $\mathrm{R}$ ) was added to $1 \mu \mathrm{l}$ of protein at the concentrations indicated in the legend to Figure 2. Cross-linking reactions (10 $\mu$ l final volume) were carried out for $1 \mathrm{hr}$ at room temperature. The reactions were quenched with $200 \mathrm{~mm}$ lysine, and the products were analyzed on a $12.5 \%$ acrylamide-SDS gel.

\section{Transfections and CATase assays}

Transfection of mouse NIH/3T3 cells and CATase assays, normalized to relative transfection efficiencies by $\beta$-galactosidase

Table 1. Synthetic oligonucleotides

\author{
BamHI-StuI linker \\ 5'-GATCCAGTGGAAGAGGAGTCCTGATCAG-3' \\ $3^{\prime}$-GTCACCTTCTCCTCAGGACTAGTC-5' \\ T7 PCR primer \\ 5'-TTGTTAATACGACTCACTATAGGGACACCATGTCTGAGACCGAGGGAAAGGCC-3 \\ fE3 primer \\ 5'-CGGAGACACCTAATATAGGCGTTCGAAGCCGTCTCATTGGTGCCGAACAGA-3' \\ fUSF primer \\ 5'-GCCTCTGTGGATTATATCCGCAAGCTTCGGCAGAGTAACCACGGCTTGTCT-3' \\ fE3-2 primer \\ 5'-GAAGCACGTCATTGTCCAGCTGCAGGTCTTTGGAGCGCTGCTGCTCCTTC-3' \\ fUSF-2 primer \\ 5'-GAAGGAGCAGCAGCGCTCCAAAGACCTGCAGCTGGACAATGACGTGCTTC-3' \\ PL-Stop primer \\ 5'-GGATCTGGGCTCTAGATTATAGTTCCTGAATTCGG-3' \\ P-Stop primer \\ 5'-GGATCTGGGCCTGTCTAGATTATTCCTGAATTCGG-3' \\ C-Stop primer \\ 5'-CGCTGCCGTCTAGAACAGTCTITGGAGCGC-3'
}


expression, were carried out as described previously (Beckmann et al. 1990).

\section{Acknowledgments}

We thank Ulrike Schindler for supplying encouragement throughout all aspects of this work. We also thank Polly Gregor and Robert Roeder for the USF expression plasmid and for discussing their results before publication. This work was supported by funds from the Howard Hughes Medical Institute / to T.K.). H.B. was supported by a grant from the Deutscher Akademischer Austauschdienst DAAD, Sonderprogramm Gentechnologie.

The publication costs of this article were defrayed in part by payment of page charges. This article must therefore be hereby marked "advertisement" in accordance with 18 USC section 1734 solely to indicate this fact.

\section{Note added in proof}

Blackwood and Eisenman (1991) have recently identified an additional b-HLH-zip protein, designated Max. They have domonstrated that Max heterodimerizes with c-Myc, L-myc, and N$m y c$ but does not heterodimerize with several other b-HLH and b-HLH-zip proteins, including AP4 and USF. In the context of the work presented here, their data would suggest that all three members of the myc family of proteins fall into the same group (i.e., they all dimerize with a common partner), although they cannot dimerize with one another. Hence, while it would appear that their respective leucine zippers are incompatible with one another, they are all compatible with the leucine zipper found in Max.

\section{References}

Ausubel, F.M., R. Brent, R.E. Kingston, D.D. Moore, J.G. Seidman, J.A. Smith, and K. Struhl. 1987. Current protocols in molecular biology. Greene Publishing Associates and Wiley Interscience, New York.

Beckmann, H., L.-K. Su, and T. Kadesch. 1990. TFE3: A helixloop-helix protein that activates transcription through the immunoglobulin enhancer $\mu \mathrm{E} 3$ motif. Genes \& Dev. 4: 167179.

Benezra, R., R.L. Davis, D. Lockshon, D.L. Turner, and H. Weintraub. 1990. The protein Id: A negative regulator of helixloop-helix DNA binding proteins. Cell 61: 49-59.

Blackwood, E.M. and R.N. Eisenman. 1991. Max: A helix-loophelix zipper protein that forms a sequence-specific DNAbinding complex with Myc. Science 251: 1211-1217.

Carr, C.S. and P.A. Sharp. 1990. A helix-loop-helix protein related to the immunoglobulin E box-binding proteins. Mol. Cell. Biol. 10: 4384-4388.

Chou, P.Y. and G.D. Fassman. 1974. Conformational parameters for amino acids in helical, $\beta$-sheets, and random coil regions calculated from proteins. Biochemistry 13: 211-222.

Davis, R.L., P.-F. Cheng, A.B. Lassar, and H. Weintraub. 1990. The MyoD DNA binding domain contains a recognition code for muscle-specific gene activation. Cell 60: 733-746.

Dang, C.V., M. McGuire, M. Buckmire, and W.M.F. Lee. 1989. Involvement of the "leucine zipper" region in the oligomerization and transforming activity of human c-myc protein. Nature 337: 664-666.

Dwarki, V.J., M. Montminy, and I.M. Verma. 1990. Both the basic region and the "leucine zipper" domain of the cyclic AMP response element binding (CREB) protein are essential for transcriptional activation. EMBO J. 9: 225-232.

Gregor, P., M. Sawadogo, and R.G. Roeder. 1990. The adenovirus major late transcription factor USF is a member of the helix-loop-helix group of regulatory proteins and binds to DNA as a dimer. Genes \& Dev. 4: 1730-1740.

Henthorn, P., M. Kiledjian, and T. Kadesch. 1990a. Two distinct transcription factors that bind the immunoglobulin enhancer $\mu \mathrm{E} 5 / \mathrm{\kappa E} 2$ motif. Science 247: 467-470.

Henthorn, P., R. McCarrick-Walmsley, and T. Kadesch. 1990b. Sequence of the cDNA encoding ITF-1, a positive-acting transcription factor. Nucleic Acids Res. 18: 677.

Horton, R.M., Z. Cai, S.N. Ho, and L.R. Pease. 1990. Gene splicing by overlap extension: Tailor-made genes using the polymerase chain reaction. Biotechniques 8: 528-535.

Hu, J.C., E.K. O'Shea, P.S. Kim, and R.T. Sauer. 1990a. Sequence requirements for coiled-coils: Analysis with $\lambda$ repressorGCN4 leucine zipper fusions. Science 250: 1400-1403.

Hu, Y-F., B. Luescher, A. Admon, N. Mermod, and R. Tjian. 1990b. Transcription factor AP-4 contains multiple dimerization domains that regulate dimer specificity. Genes \& Dev. 4: 1741-1752.

Johnson, P.F. and S.L. McKnight. 1989. Eukaryotic transcriptional regulatory proteins. Annu. Rev. Biochem. 58: 799839.

Kouzarides, T. and E. Ziff. 1989. Leucine zippers of fos, jun, and GCN4 dictate dimerization specificity and thereby control DNA binding. Nature 340: 568-571.

Landschultz, W.H., P.F. Johnson, and S.L. McKnight. 1988. The leucine zipper: A hypothetical structure common to a new class of DNA binding proteins. Science 240: 1759-1764.

Lillie, J.W. and M.R. Green. 1989. Transcriptional activation by the adenovirus Ela protein. Nature 338: 39-44.

Maniatis, T., E.F. Fritsch, and J. Sambrook. 1982. Molecular cloning: A laboratory manual. Cold Spring Harbor Laboratory, Cold Spring Harbor, New York.

Murre, C., P. Schonleber-McCaw, and D. Baltimore. 1989a. A new DNA binding and dimerization motif in immunoglobulin enhancer binding, daughterless, MyoD, and myc proteins. Cell 56: 777-783.

Murre, C., P. Schonleber-McCaw, H. Vaessin, M. Caudy, L.Y. Jan, Y.N. Jan, C.V. Cabrera, J.N. Buskin, S.D. Hauschka, A.B. Lassar, H. Weintraub, and D. Baltimore. 1989b. Interactions between heterologous helix-loop-helix proteins generate complexes that bind specifically to a common DNA sequence. Cell 58: 537-544.

Norman, C., M. Runswick, R. Pollock, and R. Treisman. 1988. Isolation and properties of cDNA clones encoding SRF, a transcription factor that binds to the c-fos serum response element. Cell 55: 989-1003.

O'Neil, K.T., R.H. Hoess, and W.F. DeGrado. 1990. Design of DNA binding peptides based on the leucine zipper motif. Science 249: 774-778.

O'Shea, E.K., R. Rutkowski, and P. Kim. 1989. Evidence that the leucine zipper is a coiled coil. Science 243: 538-542.

Patel, L., C. Abate, and T. Curran. 1990. Altered protein conformation on DNA binding by Fos and Jun. Nature 347: 572575.

Rasmussen, R.D. Benvengnu, E.K. O'Shea, P.S. Kim, and T. Alber. 1991. X-ray scattering indicates that the leucine zipper is a coiled coil. Proc. Nat1. Acad. Sci. 88: 561-564.

Shuman, J.D., C.R. Vinson, and S.L. McKnight. 1990. Evidence of changes in protease sensitivity and subunit exchange rate on DNA binding by C/EBP. Science 249: 771-774.

Smeal, T., P. Angel, J. Meek, and M. Karin. 1989. Different 


\section{Beckmann and Kadesch}

requirements for formation of Jun : Jun and Jun : Fos complexes. Genes \& Dev. 3: 2091-2100.

Talanian, R.V., C.J. McKnight, and P.S. Kim. 1990. Sequence specific binding of a short peptide dimer. Science 249: 769771.

Vinson, C.R., P.B. Sigler, and S.L. McKnight. 1989. A scissorsgrip model for DNA recognition by a family of leucine zipper proteins. Science 246: 911-916.

Voronova, A. and D. Baltimore. 1990. Mutations that disrupt DNA binding and dimer formation in the E47 helix-loophelix protein map to distinct domains. Proc. Natl. Acad. Sci. 87: 4722-4762.

Weiss, M.A., T. Ellenberger, C.R. Wobbe, J.P. Lee, S.C. Harrison, and K. Struhl. 1990. Folding transition in the DNA-binding domain of GCN4 on specific binding on DNA. Nature 347: 575-578. 


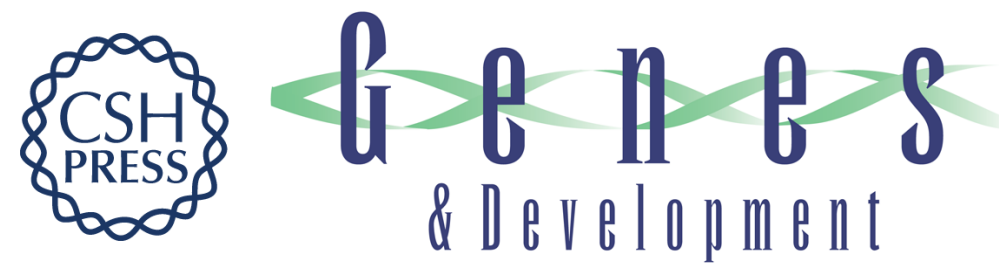

\section{The leucine zipper of TFE3 dictates helix-loop-helix dimerization specificity.}

$\mathrm{H}$ Beckmann and T Kadesch

Genes Dev. 1991, 5:

Access the most recent version at doi:10.1101/gad.5.6.1057

References This article cites 31 articles, 16 of which can be accessed free at: http://genesdev.cshlp.org/content/5/6/1057.full.html\#ref-list-1

License

Email Alerting

Service

Receive free email alerts when new articles cite this article - sign up in the box at the top right corner of the article or click here.

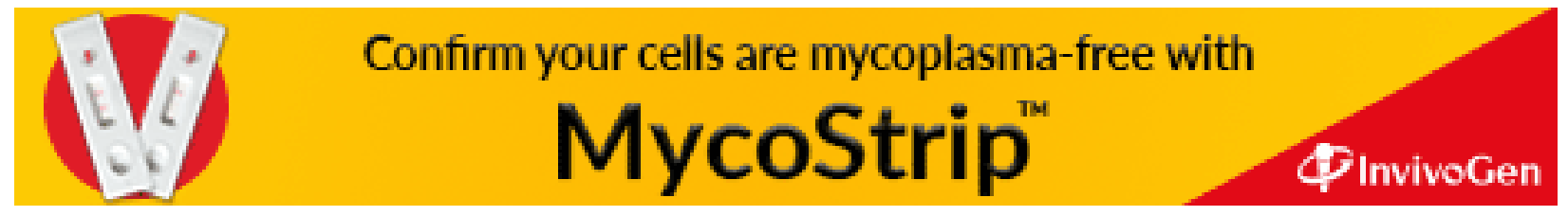

